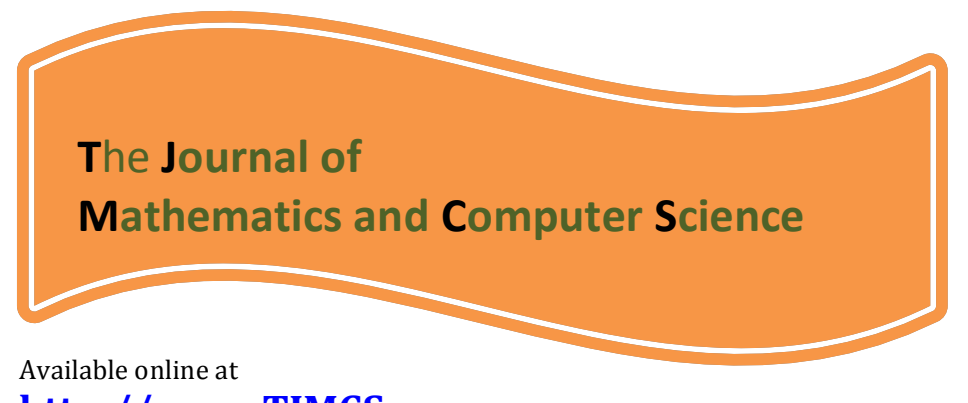

http://www.TIMCS.com

The Journal of Mathematics and Computer Science Vol. 4 No.1 (2012) 1 - 7

\title{
The Analysis of Phase Synchrony of Alpha Band Waves in Detecting the Effect of Alcohol on EEG
}

\author{
Saeed Erfanian1 , Mohammad Reza Shokoohi nia'2, Mojtaba Shokohi nia*3, \\ 1 Assistant Professor at the Faculty of Engineering of the Islamic Azad University, Garmsar Branch \\ serfanian@iau-garmsar.ac.ir \\ 2 Student of Master's Degree in Control at the Islamic Azad University, Garmsar Branch \\ mshokoohinia@yahoo.com \\ ${ }^{3}$ Lecturer at the Faculty of Control Engineering of the Islamic Azad University, Damghan Branch \\ mshokohinia@damghaniau.ac.ir
}

Received: November 2011, Revised: March 2012

Online Publication: May 2012

\begin{abstract}
Many researches have been conducted on alcoholics for predicting the probability of their addiction to alcohol as well as the effect of alcohol on EEG signals. Most of these researches study the amplitude of alpha waves and the power of signal in this band. In some of the studies, the waves so called as P300 have been studied.

In the present research paper, phase synchrony has been used as a tool for detecting alcoholics from the control group (the individuals who do not drink alcoholic beverages). The results obtained from the study indicate that in most of samples taken from the individuals who drink alcoholic beverages, there are more records of phase asynchrony between electrodes. From the results, the percentage of differentiation authenticity of about $85 \%$ was obtained for the data and a specific pair of studied canals.
\end{abstract}

Key words: Phase synchrony, alcohol dependence, Alpha band

*Corresponding author 


\section{Introduction}

Abnormal characteristics of EEG have been described extensively in many of the mental diseases including alcoholism and drug misuse. Although, there are questions about whether the statistical deviation of cerebral electric activity from the normal value is a necessary evidence for diagnosing cerebral damage or inappropriate function of brain [1], some of the statistical alterations of EEG are correlated with clinical or physiological characteristics. The reduction of alpha amplitude in frontal region is associated with anxiety and asymmetry of alpha in frontal region is dependent to depression, the increase of Teta band power is due to long consumption of marijuana, and many other similar cases have been known. It is found that some of EEG characteristics are inherited genetically which are related to the specifications of the frequency and amplitude [2].

The calculated spectrum for the absolute value of alpha power as a label for diagnosing alcohol dependence is used; in some of the tests, the dependence between the history of drinking alcoholic beverages among the members of a family and higher voltage of alpha has been seen [3]. In neurological studies such as volumetry, it is found that brain damages in alcoholics occur in frontal region; There are some other assumptions such as the scattered locations of cerebral damage or damage in the right side of the brain[4]. The analysis of phase synchrony for cerebral signals was firstly used by Lachaux; it was shown that this analysis is suitable for epilepsy and studies on insanity and is an appropriate method for understanding the process of memory function [5]. In the present study, the analysis of phase synchrony is used for detecting the alcoholics and control group which may be employed for designing a cheap and portable distinguishing system. The calculation of the characteristic of amplitude in frequency domain that is called power spectrum is carried out by statistical method including periodogram and maximum entropy. While signal phase contains a great section of data which process the signal behavior such as the procedure of signal changes in time and it may be used as an important characteristic in diagnosis and treatment, it is neglected in such processes.

\section{Methodology}

\section{2-1. Studied Data}

EEG data studied in the present research paper was collected for doing an extensive test on EEG correlation with primary genetic desire to alcoholism. The data was recorded by 64 electrodes on scalp for one second after being stimulated by a set of images from two participating groups including the alcoholics and control group with sampling frequency of $256 \mathrm{~Hz}$. These data belongs to the neurodynamic laboratory of the State New York Health Center University. This recording process has been conducted 120 times for any of 122 participants. 


\section{2-2. EEG Pre-Processing: Separation of Alpha Band}

One pair of centrifugal filter with finite impact response (FIR) and Parzen weighty window which has a cross band of $8-12 \mathrm{~Hz}$ and pass band with a width of $2 \mathrm{~Hz}$ and enjoys a free-distortion linear phase response, illustrated in Figure I, was used for separating waves in alpha rhythm from EEG signal.

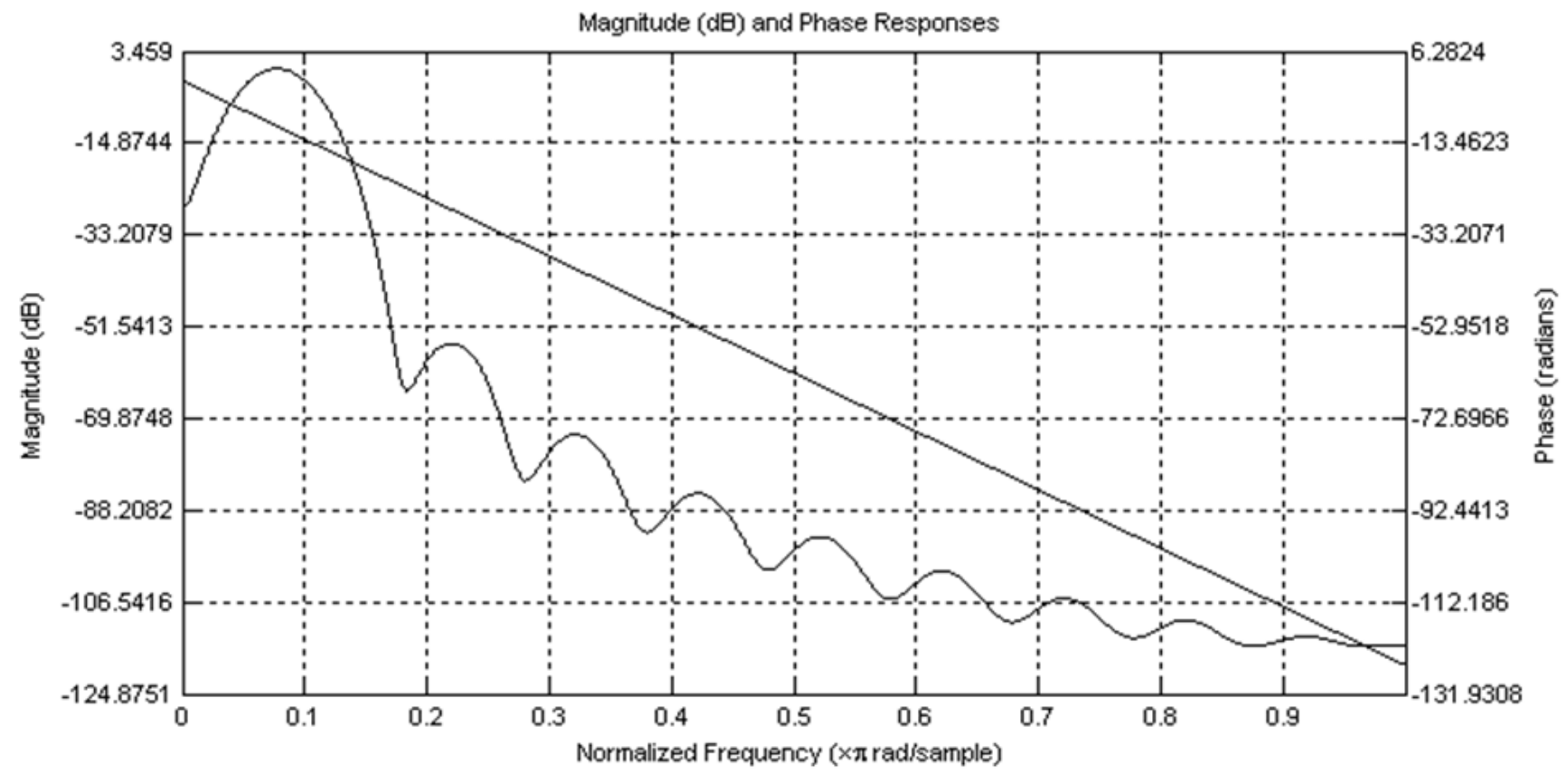

Figure I. Phase response and filter amplitude used for separating alpha rhythm.

\section{2-3. Analytic Signal}

For calculating the phase difference among electrodes from this block, firstly, Hilbert signal transformation is computed, then, it is multiplied by $(\mathrm{j}=\sqrt{-1})$ and added with the primary signal; thus the complex signal is calculated. Hilbert signal transformation is indeed the calculation of signal convolusion or $1 / \pi \mathrm{t}$. Consequently, the convolusion properties of Fourier transformation $\mathrm{SH}(\mathrm{W})$ is equal to the product of Fourier transformation $s(t)$ and is $1 / \pi t$. For the frequencies in conformity with the physical phenomena

$\mathrm{S}(\mathrm{w})=-\mathrm{iSH}(\mathrm{w}), \mathrm{w}>0$. This indicates that Hilbert transformation is calculable in all frequencies by an ideal filter with a single amplitude response and a constant (fixed) phase response with a delay of $\pi / 2$.[6]. The calculation of complex signal in this research is done by analytic signal block in simulink (Figure II). 


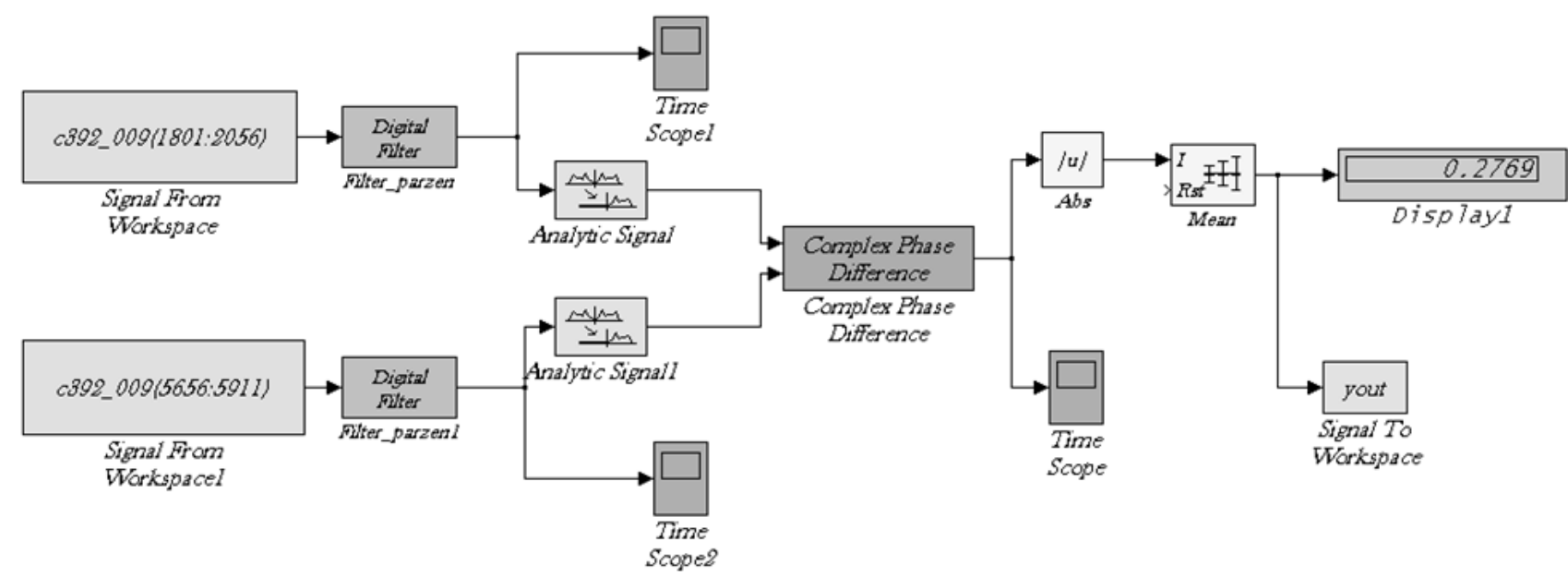

Figure II. Simulink model designed for calculating the time average of phase difference in a pair of electrodes

\section{2-4. EEG Processing: Phase Synchrony}

After obtaining the complex signal, the instantaneous phase difference for any pair of electrode is calculated for all samples by the block of Complex Phase Difference. Then, its absolute value and subsequently the statistical average of the phase difference is calculated by the next block.

\section{2-5. Selection of Suitable Electrodes and Alpha Rhythm}

The statistical characteristic used for classification in this research is the time average of phase difference of a pair of electrodes. The first and most important part was selecting appropriate electrodes (concerning the highest difference between two groups). Two different assumptions may be considered as the starting point for finding the desired electrodes. The first theory is that if the under-study electrodes are in one region, in this case, the effect of further sources on them will be the same and it is expected that they enjoy more phase synchrony because the electrodes are near each other; in such a case, the phase difference occurred due to alcohol consumption will be more detectable. The other starting point is based on this principle that those electrodes are chosen in which the amplitudes of their alpha bands are more than others because in most of conducted researches, it is evident that the amplitude of alpha wave alters due to alcohol consumption.

\section{Results}

Although it seems that it is sufficient to study the phase synchrony of a pair of electrodes in occipital region for selecting appropriate electrodes, but, for being sure of the most probable selection, in an initial test, the time average of phase difference of all electrodes was calculated for one of the data obtained from alcoholics and control group; this was 
done bipolar (without selecting reference electrode and for all probable couples of electrodes) and the pair of electrodes that enjoy more suitable differentiation were chosen for the next stage from which we may refer to C3-OZ, C4-OX, C3-P4, C4-P4, P0801, C4-02, C4-P4, Oz-01, and .... After studying more data, it was found that there is more difference between data of two groups for two pairs of electrodes (01-02, P08-C4). Also, in general, the value of phase difference for the data obtained from alcoholics was more than the control group. Considering the low average of phase difference for alcoholics and P08-01 which is 11.radian and its calculation for 60 items of data which have been selected randomly, the percentage of differentiation authenticity was obtained about $85 \%$ and the results have been illustrated in the diagram of Figure III but assuming the border value of 19.0 radian and C4-02 electrodes no better result than \%67.75 was gained.

\begin{tabular}{|c|c|}
\hline alcohol & control \\
\hline $0.1502-+0.369$ & $0.863-+0.0328$ \\
\hline
\end{tabular}

Table 1: Values of phase difference for control group and alcoholics $\mathrm{P}<0.001$.

\section{Discussion \& Conclusion}

The analysis of changes of a signal due to its nature of changeability with time and its dynamic behavior may employ new characteristics and obtain results through amplitude processing which deems impossible. The alterations of cerebral signals during time are so great that they are sometimes called as semi-noise. Since the source of recorded signals through different electrodes is the same, but, the amplitudes of recorded signals are not the same because of heterogeneity and non-isotopic volumetric conductor of head.

In the present research, the results indicate that there is a significant difference between two groups concerning the value of phase synchrony in P08 and 01 electrodes which is shown in table I.

It is suggested that some processing such as calculation of fractal dimension and modeling of alterations as well as analyzing the physiologic effects lead to creating difference between those two groups will be conducted in the future.

Although employing phase synchrony in processing bio-signals may be helpful in a proper diagnosis, but attention should be paid that in pre-processing such as filtering, undesired alterations do not occur in phase of signal. 


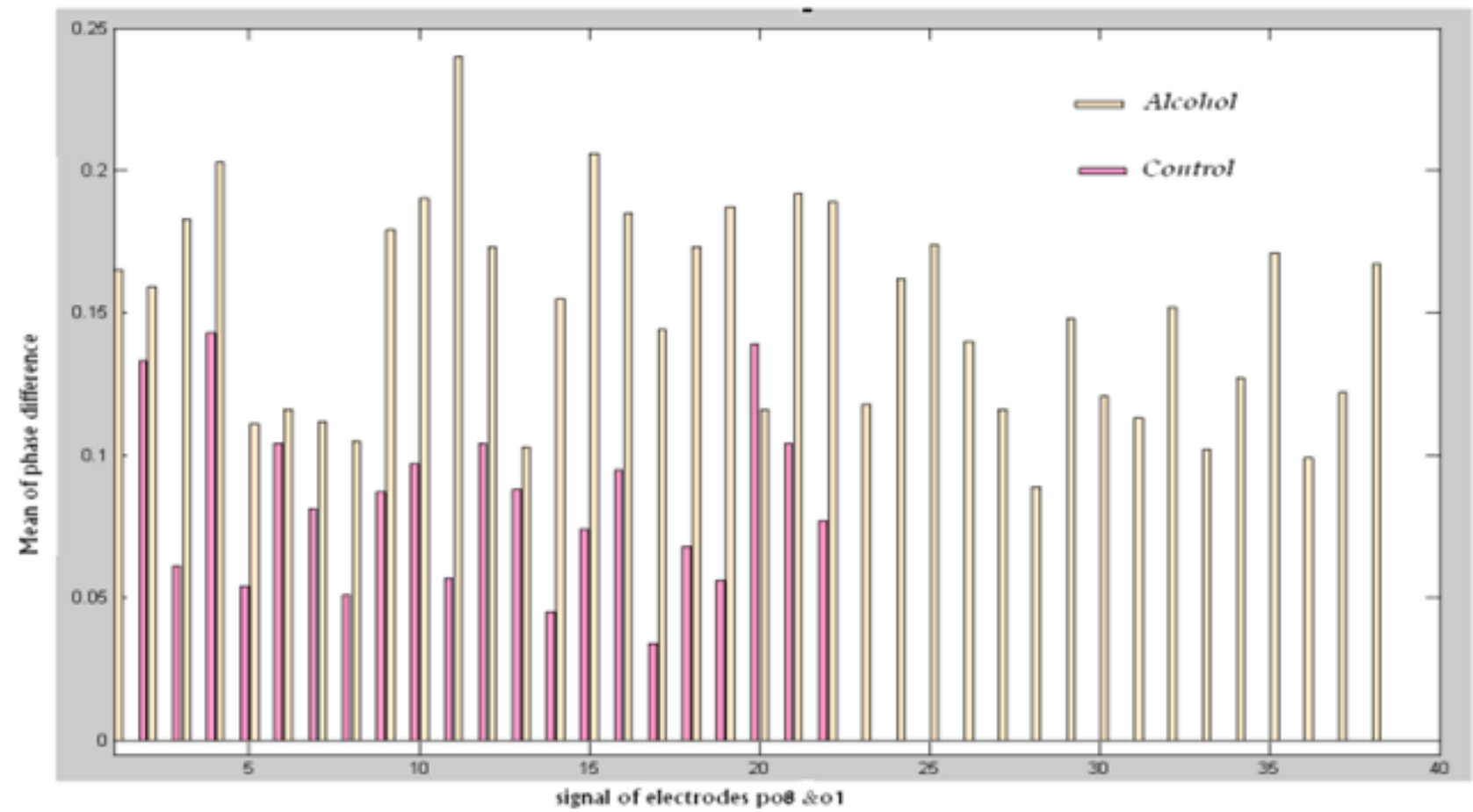

Figure III. The diagram of the average of phase difference between P08 and 01 electrodes in control group and patients

\section{References}

[1]Pedro Coutin-Churchman R.o.M., YelitzaAñez , Fa'timaVergara Clinical correlates of quantitative EEG alterations in alcoholic patients, Clinical Neurophysiology 117 (2006)12.

[2]Cindy L .Ehlers E.P., Association of EEG alpha variants and alpha power with alcohol dependence in Mexican American young adults, Alcohol41(2007)8.

[3]Eveline A.de Bruin S.B., Cornelis J .Stam, Koen B.E.Bo“cker „J J.Leon Kenemans M.N.V., Abnormal EEG synchronisation in heavily drinking students, Clinical Neurophysiology 115(2004)8.

[4]Cindy L .Ehlers E.P., Marc A .Schuckit, EEG alpha variants and alpha power in Hispanic American and white non-Hispanic American young adults witha family history of alcohol dependence, Alcohol 41 (2008)8. 
[5]Gleb V .Tcheslavski A.A.L.B., Phase synchrony and coherence analyses of EEG as tools to discriminate between children with and without attention deficit disorder, Biomedical Signal Processing and Control 1 (2006)11.

[6]Michael Rosenblum A.P., J"urgenKurths , CarstenSch"afer P.A.T., Phase synchronization :from theory to data analysis, Handbook of Biological Physics 4 (2001) 